\title{
An Uncommon Presentation of Progressive Supranuclear Palsy Diagnosed on DaT Scan
}

\author{
Victoria Lord, Somnath Sinha ${ }^{2}$ \\ 1. 2. General Medicine, Ipswich Hospital, QLD, Australia
}

$\square$ Corresponding author: Subhasish Bose, drsbose@gmail.com

Disclosures can be found in Additional Information at the end of the article

\section{Abstract}

Diagnosis of Parkinson's disease can be difficult in presence of atypical symptoms and signs. While the commonest reason of parkinsonism is idiopathic Parkinson's disease (PD), other disease entities that can mimic PD should be considered in the light of clinical history and examination findings. However, still some diagnostic dilemmas may exist which may need special investigative modalities. In recent years, advancements have been made to use single proton emission computed tomography (SPECT) scan to determine the cause of parkinsonian features. However, clinical use of this specialized tool is still not well-recognized. We have presented here a case of supranuclear palsy that was difficult to diagnose clinically and had appropriate final diagnosis with the help of dopamine transporters (DaT) scan.

Categories: Internal Medicine, Pathology

Keywords: diagnosis, spect, parkinson's disease, palsy, dat scan, tremor

\section{Introduction}

Diagnosis of Parkinson's disease can be difficult in presence of atypical symptoms and signs. While the commonest etiology of parkinsonism is idiopathic Parkinson's disease (PD), other disease entities that can mimic PD should be considered in light of clinical history and examination findings. However, still some diagnostic dilemmas may exist which may need special investigative modalities. In recent years, advancements have been made with regards to use of single proton emission computed tomography (SPECT) scan to determine the cause of parkinsonian features. However, clinical use of this specialized tool is still not well-recognized. We have presented here a case of progressive supranuclear palsy that was difficult to diagnose clinically and had appropriate final diagnosis with the help of dopamine transporters (DaT) scan.

\section{Case Presentation}

\section{Published 05/14/2011}

\section{(c) Copyright 2011}

Lord et al. This is an open access article distributed under the terms of the Creative Commons Attribution License CC-BY 3.0., which permits unrestricted use, distribution, and reproduction in any medium, provided the original author and source are credited.
A 44-year-old male with learning difficulties was admitted from a rehabilitation unit with a history of being mugged following inappropriate behavioral outburst. His caregivers were worried that over the last six months he had difficulty swallowing, inappropriately urinated, and was extremely lethargic and slept during the day.

There was no other significant past history or any fluctuations in his physical or mental condition. He was on quietapine and prochlorperazine for the last one year. Although these medicines were stopped owing to his new symptoms, his clinical features did not improve. There was no known drug allerg or the use of any recreational drugs or alcohol. He had no immediate family, having lived in an orphanage in the early days, and had no formal schooling. 


\section{Cureus}

Examination was limited due to his natural apathy and refusal to be examined formally for any length of time. He was emaciated and dribbled of saliva, incontinent of urine and had postural hypotension. Neurological examination revealed hypomimia with reduced blink rate and generalized rigidity with a left foot dystonia. The eye movements were restricted for both vertical and horizontal gaze. The rest of the examination was inconclusive. Psychometric tests and tests for memory or mood was not possible.

Blood tests, including complete blood count, basic metabolic panel, liver function test, glucose, thyroid function test, anti-thyroid peroxidase antibody, serum B12, folic acid, serum iron, ferritin, testosterone, serum copper, trepanoma pallidum hemagglutination test (TPHA), HIV antibody, and short synacthen test, came back as normal/negative. Urine analysis was normal. CSF examination was normal. Chest X-ray, CT brain scan and subsequent MRI of the brain were reported as normal.

At this point, the differential diagnoses included drug-induced Parkinsonism, idiopathic Parkinson's disease (PD), essential tremor, or Parkinson's Plus Syndrome.

$\mathrm{A}^{123}$ I FP-b-CIT SPECT dopamine transporter scan (Figure 1) was done. It showed bilateral reduced uptake of tracer. Taking into account the other clinical features, the diagnosis of progressive supranuclear palsy (PSP) was confirmed.

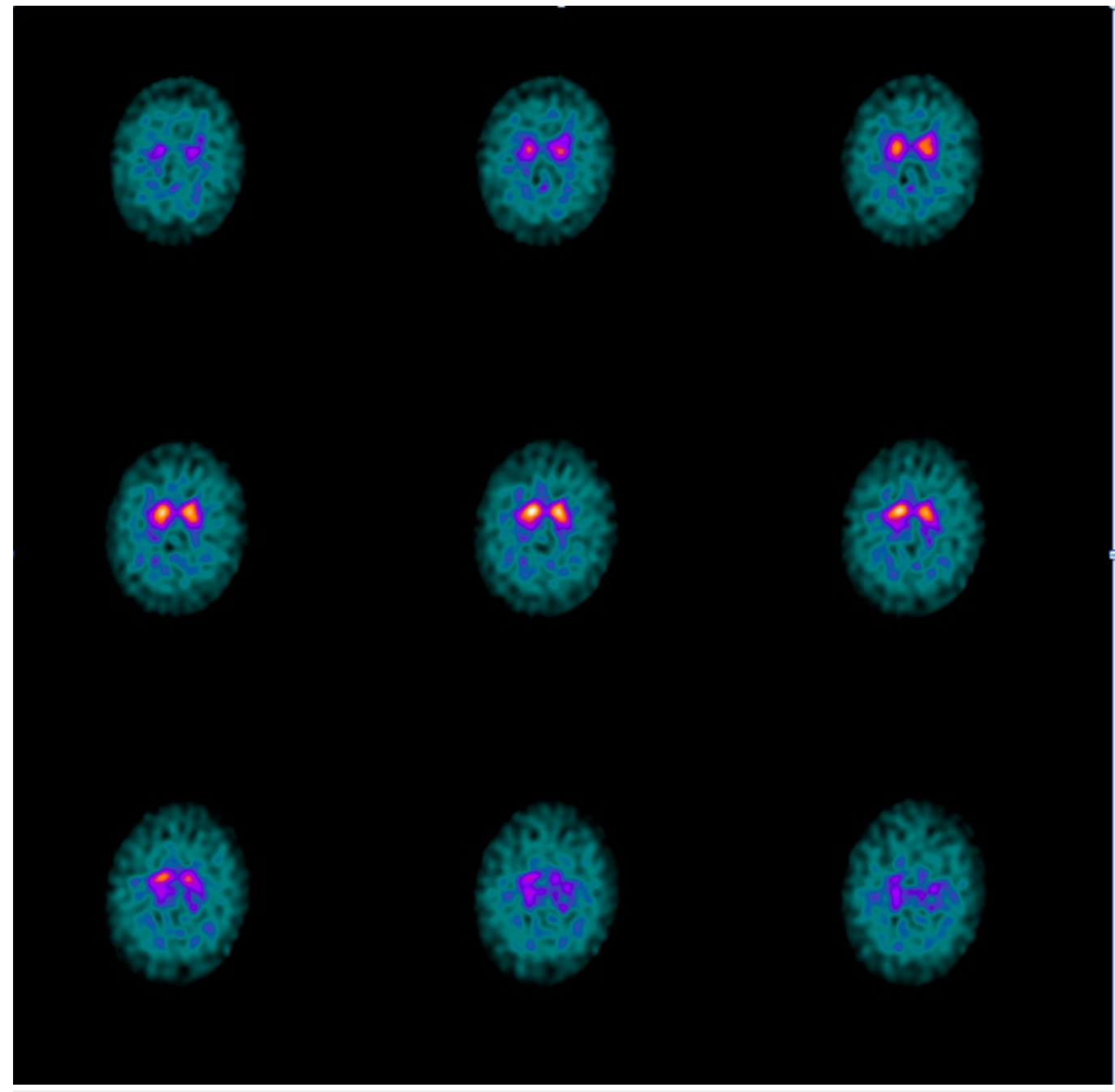

FIGURE 1: SPECT imaging series of our patient showing 
Please compare with Figure 2.

\section{Discussion}

PSP is an uncommon parkinsonian syndrome that can mimic PD in its early phase. It is also known as Steele Richardson Olszewski syndrome. Apathy, disinhibition, dysphoria, and anxiety are common features of PSP. Characteristic clinical signs include a vertical supranuclear palsy with downward gaze abnormalities and postural instability with unexplained falls [12]. Bradykinesia and rigidity are typically symmetrical in onset. The typical rest tremor of PD is rare in PSP [3]. A pseudobulbar palsy with associated dysarthria and dysphagia develops in approximately 80 percent or more of patients with PSP. Although the cognitive impairment may be mild in the first few years after clinical disease onset, majority of patients develop a frontal lobe-like dementia syndrome [4]. In PSP, the response to levodopa is typically poor, but about $20 \%$ of patients have some improvement initially or in the early stages of the disease [1]. The overall prognosis of PSP is poor because of its progressive nature, with death occurring at a median of six years after onset of disease [5].

SPECT is a nuclear medicine technique in which temporal changes in the concentrations of radioactive tracers are recorded in brain. Both pre- and post-synaptic dopaminergic functions can be measured with use of specific tracers. Several radioligands have been studied and developed for SPECT that can be used as sensitive and quantitative presynaptic dopaminergic markers. These are based on binding to the dopamine transporter (DaT) or the vesicular monoamine transporter Type 2 (VMAT2) or on measurement of the aromatic amino acid decarboxylase activity. The tracers of dopamine pre-synaptic reuptake sites (also called DaT sites) were developed more recently. In recent years, ${ }^{123}$ I FP-b-CIT SPECT is mainly used as a selective tropane that is a cocaine analog. SPECT imaging is obtained after a specific time period from the time of injection of the tracer and images are acquired using a gamma camera. DaT scan images are interpreted visually, based on the appearance of the striata. Strict guidelines have been discussed in scientific literature that suggests that the reconstruction image pixel size should be between 3.5 and $4.5 \mathrm{~mm}$ with slices 1 pixel thick. The image should be interpreted in transaxial slices parallel to the anterior commissure-posterior commissure line. Whether an image is normal or abnormal is determined by assessing the extent (as indicated by the shape) and intensity of the striatal signal. Image interpretation does not involve integration of the striatal image appearance with clinical signs and/or symptoms. The interpretation of DaT scan can be made semi-quantitative by using predefined region of intererst (ROI) on the brain scan. In DaT scan, the radiotracer (Ioflupane $-{ }^{123} \mathrm{I}$ ) binds to the Dopamine Transporter (DaT) molecule and normally looks like a comma (Figure 2) on scanning of the brain reflecting the anatomical areas of putamen and caudate nuclei [6]. In Idiopathic PD and Parkinson's Plus Syndrome, the radiotracer uptake is diminished starting from the putamen and hence looks like a full stop in the advanced stage. Although DAT scan cannot differentiate Idiopathic PD from Parkinson's Plus Syndrome (eg, Multisystem Atrophy, Lewy body dementia, PSP), it is normal in essential tremor and drug-induced Parkinsonism [7]. 


\section{Cureus}

FIGURE 2: A normal brain scan with the SPECT imaging showing normal dopamine transporter (DaT) activity.

Our patient had history suggestive of new onset pseudobulbar palsy, worsening of his baseline cognitive skills and behavioral changes. Clinical examination was significant for gaze abnormality, autonomic dysfunction and odd behavior. Given the history of recent use of medication that can cause drug-induced Parkinsonism, it was important to determine the exact nature of this patient's underlying neurological problem before putting him on any potentially 
long-term medication therapy which may have toxic adverse effects. While drug-induced Parkinsonism is usually reversible, at least $10 \%$ cases of persistent Parkinsonism have been reported despite drug withdrawal [8]. It has been unclear if this persistence is due to ongoing toxicity of the responsible drug or to the presence of an underlying Parkinsonian syndrome. The known risk factors for drug-induced Parkinsonism are: older age, female sex, higher dose as well as rate of dose escalation of offending drugs, pre-existing extra-pyramidal disorder, dementia, HIV infection and familial PD [9]. The abnormal DaT scan virtually excluded the possibility of drug-induced Parkinsonism and therefore we were confident to start anti-PD medications on the patient with a diagnosis of PSP.

The patient was treated with transdermal rotigotine (dopamine agonist), stalevo (Levodopa/ Entacapone combination), transdermal rivastigmine (AntiCholineEsterase Inhibitor), and quietapine. He had modest improvement in both motor and non-motor symptoms over the following twelve months, regained body weight and participated voluntarily with ongoing behavioral psychotherapy.

\section{Conclusions}

Although its clinical use is still not well-recognized, advances in single proton emission computed tomography (SPECT) scanning now enable the causation of parkinsonian features to be determined in many circumstances. We present here a case of supranuclear palsy that was difficult to diagnose clinically but that had appropriate final diagnosis with the help of a dopamine transporters (DaT) scan.

\section{Additional Information Disclosures}

Human subjects: All authors have confirmed that this study did not involve human participants or tissue. Conflicts of interest: In compliance with the ICMJE uniform disclosure form, all authors declare the following: Payment/services info: All authors have declared that no financial support was received from any organization for the submitted work. Financial relationships: All authors have declared that they have no financial relationships at present or within the previous three years with any organizations that might have an interest in the submitted work. Other relationships: All authors have declared that there are no other relationships or activities that could appear to have influenced the submitted work.

\section{References}

1. Litvan I, Campbell G, Mangone CA, et al: Which clinical features differentiate progressive supranuclear palsy (Steele-Richardson-Olszewski syndrome) from related disorders? A clinicopathological study. Brain. 1997, 120:65. 10.1093/brain/120.1.65

2. Litvan I, Agid Y, Jankovic J, et al.: Accuracy of clinical criteria for the diagnosis of progressive supranuclear palsy (Steele-Richardson-Olszewski syndrome). Neurology. 1996, 46:922.

3. Quinn N: Parkinsonism--recognition and differential diagnosis. BMJ. 1995, 310:447. 10.1136/bmj.310.6977.447

4. Verny M, Jellinger KA, Hauw JJ, et al.: Progressive supranuclear palsy: a clinicopathological study of 21 cases. Acta Neuropathol. 1996, 91:427. 10.1007/s004010050446

5. Maher ER, Lees AJ: The clinical features and natural history of the Steele-RichardsonOlszewski syndrome (progressive supranuclear palsy). Neurology. 1986, 36:1005.

6. Brucke T, Asenbaum S, Pirker W, et al.: Measurement of the dopaminergic degeneration in Parkinson's disease with [123I] beta-CIT and SPECT. Correlation with clinical findings and comparison with multiple system atrophy and progressive supranuclear palsy. J. Neural Transm Suppl. 1997, 50:9-24.

7. Castrejon A S, Vicente A M G, et al.: 123-I Ioflupane presynaptic nigrostriatal imaging in patients with movement disorders. Brazilian Archives of Biology and Technology. 2005, 48: 


\section{Cureus}

8. Mena MA, de Yébenes JG: Drug-induced parkinsonism. Expert Opin Drug Saf. 2006, 5:759. $10.1517 / 14740338.2013 .787065$

9. Alvarez MV, Evidente VG: Understanding drug-induced parkinsonism: separating pearls from oysters. Neurology. 2008, 70:32-4. 